\title{
Safinamide Modulates Striatal Glutamatergic Signaling in a Rat Model of Levodopa-Induced Dyskinesia
}

\author{
F. Gardoni, M. Morari, J. Kulisevsky, A. Brugnoli, S. Novello, C. A. Pisanò, C. Caccia, \\ M. Mellone, E. Melloni, G. Padoani, V. Sosti, S. Vailati, and C. Keywood
}

Department of Pharmacological and Biomolecular Sciences, University of Milan, Milan, Italy (F.G., M.Me.); Department of Medical Sciences, Section of Pharmacology, University of Ferrara, Ferrara, Italy (M.Mo., A.B., S.N., C.A.P.); Sant Pau Institute of Biomedical Research, Barcelona, Spain (J.K.); Universitat Autònoma de Barcelona, Universitat Oberta de Catalunya, Barcelona, Spain (J.K.); Centro de Investigación Biomédica en Red sobre Enfermedades Neurodegenerativas, Madrid, Spain (J.K., V.S.); Department of R\&D, Zambon SpA, Bresso, Milan, Italy (C.C., E.M., G.P., S.V., C.K.); and Neuropsychopharmacology Laboratory, Movement Disorders Unit, Hospital de la Santa Creu i Sant Pau, Barcelona, Spain (V.S.)

Received June 24, 2018; accepted September 17, 2018

\begin{abstract}
Safinamide (Xadago) is a novel dual-mechanism drug that has been approved in the European Union and United States as add-on treatment to levodopa in Parkinson's disease therapy. In addition to its selective and reversible monoamine oxidase $B$ inhibition, safinamide through use-dependent sodium channel blockade reduces overactive glutamatergic transmission in basal ganglia, which is believed to contribute to motor symptoms and complications including levodopa-induced dyskinesia (LID). The present study investigated the effects of safinamide on the development of LID in 6-hydroxydopamine (6-OHDA)-lesioned rats, evaluating behavioral, molecular, and neurochemical parameters associated with LID appearance. 6-OHDA-lesioned rats were treated with saline, levodopa $(6 \mathrm{mg} / \mathrm{kg})$, or levodopa
\end{abstract}

plus safinamide $(15 \mathrm{mg} / \mathrm{kg})$ for 21 days. Abnormal involuntary movements, motor performance, molecular composition of the striatal glutamatergic synapse, glutamate, and GABA release were analyzed. In the striatum, safinamide prevented the rearrangement of the subunit composition of $N$-methyl-Daspartate receptors and the levodopa-induced increase of glutamate release associated with dyskinesia without affecting the levodopa-stimulated motor performance and dyskinesia. Overall, these findings suggest that the striatal glutamate-modulating component of safinamide's activity may contribute to its clinical effects, where its long-term use as levodopa add-on therapy significantly improves motor function and "on" time without troublesome dyskinesia.

\section{Introduction}

Safinamide (Xadago) is a novel drug approved in the European Union and United States as an add-on to levodopa for the treatment of mid-to-late stage fluctuating patients with idiopathic Parkinson's disease (PD). Beyond selective and reversible monoamine oxidase $\mathrm{B}$ (MAO-B) inhibition (Caccia et al., 2006), safinamide is endowed with nondopaminergic properties including use-dependent voltage-operated sodium channel blockade, which results in the inhibition of in vivo glutamate (Glu) release (Morari et al., 2018).

Long-term treatment with levodopa still represents the most effective therapy for motor symptoms relief in PD patients. However, motor fluctuations and involuntary movements known as levodopa-induced dyskinesia (LID) represent the major side effects (Bastide et al., 2015). Several neurochemical and molecular changes that are thought to underlie

This work was supported by Zambon S.P.A

F.G. and M.M. contributed equally to this work.

https://doi.org/10.1124/jpet.118.251645.
LID have been reported, including alterations in glutamatergic transmission (Cenci and Lindgren, 2007). Chronic treatment with high levodopa doses $(100 \mathrm{mg} / \mathrm{kg})$ led to an increase in basal extracellular Glu levels in the striatum and substantia nigra reticulata $(\mathrm{SNr})$ of 6 -hydroxydopamine (6-OHDA) hemilesioned dyskinetic rats (Robelet et al., 2004). However, further studies with lower doses of chronic levodopa found either a reduction (12 mg/kg; Dupre et al., 2011) or no change $(4 \mathrm{mg} / \mathrm{kg}$; Nevalainen et al., 2013) in baseline Glu levels in the dopamine (DA)-depleted striatum of dyskinetic rats. Moreover, microdialysis performed in awake dyskinetic rats (Dupre et al., 2011; Paolone et al., 2015) and mice (Brugnoli et al., 2016) revealed that administration of a challenge dose of levodopa $(6 \mathrm{mg} / \mathrm{kg})$ caused the appearance of abnormal involuntary movements (AIMs), and simultaneously evoked Glu levels in the DA-depleted striatum. Interestingly, compounds able to reduce LID also normalized striatal Glu (Dupre et al., 2011; Paolone et al., 2015), suggesting that the increase of striatal Glu release detected during microdialysis experiments might be instrumental in LID appearance. Elevation of dialyzed Glu levels following levodopa challenge was also observed in the

ABBREVIATIONS: AIM, abnormal involuntary movement; ALO, axial, limbic, and orolingual; AMPAR, $\alpha$-amino-3-hydroxy-5-methyl-4isoxazolepropionic acid receptor; ANOVA, analysis of variance; DA, dopamine; Glu, glutamate; LID, levodopa-induced dyskinesia; MAO-B, monoamine oxidase B; NMDAR, N-methyl-D-aspartate receptor; 6-OHDA, 6-hydroxydopamine; PD, Parkinson's disease; PKA, protein kinase A; PSD, postsynaptic density; SNr, substantia nigra reticulata; SPNs, striatal projection neurons; TIF, Triton X-100-insoluble fraction. 
SNr but not globus pallidus of dyskinetic rats (Bido et al., 2011; Mela et al., 2012; Paolone et al., 2015) and mice (Bido et al., 2015; Brugnoli et al., 2016). In SNr, elevation of Glu was associated with elevation of GABA, which was proven to be an index of activation of the striato-nigral direct pathway in LID (Mela et al., 2007, 2012; Bido et al., 2011; Marti et al., 2012; Paolone et al., 2015). Accordingly, all of the aforementioned studies indicate that agents restoring dopaminergic tone and normalizing excessive Glu release hold promise in controlling motor symptoms and motor complications in PD.

Chronic levodopa administration also induces adaptive modifications at synapses of striatal projection neurons (SPNs). These include an aberrant synaptic localization of $N$-methyl-D-aspartate-type Glu receptors, which are correlated with the alteration of striatal synaptic plasticity (Calabresi et al., 2016). In particular, $N$-methyl-D-aspartate receptor (NMDAR) overactivation, paralleled by an altered synaptic distribution of specific NMDAR subtypes (Gardoni et al., 2006, 2012; Mellone et al., 2015), plays a role in PD and LID (Mellone and Gardoni, 2018). Indeed, after chronic levodopa treatment, the GluN2B subunit is aberrantly distributed to the extrasynaptic membrane in the striatum of dyskinetic rats, while the synaptic levels of GluN2A are augmented (Gardoni et al., 2012; Stanic et al., 2017). In addition, a significant increase in the GluN2A/GluN2B subunit ratio at synapses has been confirmed in dyskinetic monkeys as well as in dyskinetic PD patients (Mellone et al., 2015), indicating that this synaptic trait is a marker of LID. This event is correlated with an increased interaction of the GluN2A subunit with scaffolding proteins of the excitatory synapse (Gardoni et al., 2006; Mellone et al., 2015). Conversely, several reports did not observe alterations of striatal NMDAR expression levels in both animal models (Calon et al., 2002; Gardoni et al., 2006; Mellone et al., 2015) and patients (Mellone et al., 2015).

Alterations in $\alpha$-amino-3-hydroxy-5-methyl-4-isoxazolepropionic acid (AMPA) Glu receptor synaptic localization and phosphorylation have been also documented in LID models and PD patients. Enhanced phosphorylation of the GluA1 subunit on serine 845 by protein kinase A (PKA) has been described in rodent and nonhuman primate models of LID (Santini et al., 2007, 2010; Errico et al., 2011). Importantly, increased phosphorylation of this phosphosite has been associated with synaptic expression of AMPA receptors (AMPARs), and it has been defined as a marker of LID.

The aim of this study was to investigate the effects of safinamide on the development of LID in 6-OHDA hemilesioned rats. Behavioral (AIMs and motor performance), molecular (NMDAR and AMPAR subunits), and neurochemical (Glu and GABA release) parameters were evaluated in dyskinetic rats acutely challenged with levodopa.

\section{Materials and Methods}

Animals. Adult male Sprague-Dawley rats $(n=180 ; 150-175$ g; Charles River Laboratories, Calco, Italy) were used in this study. Rats were housed in the animal facilities at the University of Ferrara $(n=$ $60)$, University of Milan $(n=60)$, or Research Institute Sant Pau Hospital $(n=60)$ throughout the study. Animal handling and all procedures were carried out in accordance with the guidelines for the use of the laboratory animals in biomedical research provided by the European Union's directives (2010/63EU) and Italian (D.lgs. 26/2014 and 714/2016-PR) and Spanish Laws.
Unilateral 6-OHDA Rat Model. Animals were unilaterally injected (stereotaxic coordinates from bregma and the dural surface: antero-posterior $=-4.4$, lateral $=1.2$, dorso-ventral $=-7.8$, tooth bar at $-2.4 \mathrm{~mm}$ ) (Paxinos and Watson, 1986) with $12 \mu \mathrm{g} \mathrm{6-OHDA}$ hydrobromide in the (right) medial forebrain bundle. Two weeks later, the degree of DA depletion was evaluated by counting the number of contralateral rotations in response to a test dose of apomorphine $(0.05 \mathrm{mg} / \mathrm{kg}$, subcutaneous injection; dissolved in $0.1 \%$ ascorbate saline and administered at the volume of $1 \mathrm{ml} / \mathrm{kg}$ body weight) for 40 minutes. Animals able to perform at least 200 contralateral turns were included in the study. This number of contralateral turn behavior is associated with a reduction of $>95 \%$ of striatal dopaminergic fibers (Paillé et al., 2010).

Pharmacological Treatment. Two weeks after apomorphine injection, rats were randomly assigned to three different groups, chronically treated (1 injection/d for 21 days) with: 1) levodopa $(6 \mathrm{mg} / \mathrm{kg}$; dissolved in saline and administered at the volume of $1 \mathrm{ml} / \mathrm{kg}$ body weight) plus benserazide (12 $\mathrm{mg} / \mathrm{kg}$; dissolved in saline) subcutaneously administered, 2) saline subcutaneously administered, and 3) levodopa/benserazide (as previously described) plus safinamide $(15 \mathrm{mg} / \mathrm{kg}$ i.p., dissolved in saline and administered at the volume of $1 \mathrm{ml} / \mathrm{kg}$ body weight 30 minutes before levodopa/benserazide).

Behavioral Observations. Levodopa-induced AIMs were evaluated on days 1, 6, 11, 16, and 21 during treatment (see Fig. 1) using a highly validated AIM scale for rats (Cenci et al., 1998; Cenci and Lundblad, 2007). Briefly, rats were observed individually for 1 minute every 20 minutes during 3 hours after levodopa injection. AIMs affecting head, trunk, and forelimb were classified according to their topographic distribution in four subtypes: 1) axial AIMs, twisted posture or choreiform twisting of the neck and upper body toward the side contralateral to the lesion; 2) forelimb AIMs, jerky or dystonic movement of the contralateral paw; 3) orolingual AIMs, orofacial muscle twitching, empty masticatory movements, and contralateral tongue protrusion; and 4) locomotive AIMs (i.e., contralateral turning). Each subtype was scored on a severity scale from 0 to 4 , where $0=$ absent, 1 = present during less than half of the observation time $(<30$ seconds), $2=$ present for more than half of the observation time ( $>30$ seconds), $3=$ present all of the time (= 1 minute) but suppressible by external stimuli, and $4=$ present all of the time and not suppressible by external stimuli. The total AIM score for each test session was obtained by summing the scores of all observation time points. Axial, forelimb, and orolingual AIMs were presented collectively, as the global [i.e., axial, limbic, and orolingual (ALO)] AIM score. Conversely, locomotive AIMs were analyzed as a separate item, given that they differ greatly from the other three subtypes in terms of pharmacological features, anatomic substrates, and pathophysiological significance. To explore whether the potential antidyskinetic effect of safinamide is not due to primary motor inhibition, the rotarod performance was evaluated before levodopa administration and 60 minutes post-levodopa (i.e., at peak dyskinesia) administration using a fixed-speed protocol (Marti et al., 2012; Paolone et al., 2015). Rotarod performance was first assessed before starting the 21-day course of levodopa administration (animals were trained 5 days on the rotarod until their performance was reproducible) and then assessed during levodopa treatment at days 3, 8, 13, and 18 (see Fig. 1).

In Vivo Microdialysis: GABA and Glu Release. Twenty-four hours after the end of chronic treatment (day 22), dyskinetic rats were stereotaxically implanted, under isoflurane anesthesia, with one microdialysis probe in the DA-depleted dorsolateral striatum and another in the ipsilateral SNr, as previously described (Morari et al., 1996). Coordinates from bregma and dural surface (Paxinos and Watson, 1986) were (in millimeters): striatum, antero-posterior + 1.0 , medio-lateral \pm 3.5 , and dorso-ventral -6.0 ; and $\mathrm{SNr}$, anteroposterior -5.5 , medio-lateral \pm 2.2 , and dorso-ventral -8.3 . Concentric microdialysis probes were constructed using AN69 (Gambro Industries, Meyzieu, France) semipermeable hollow membranes (65 kDa molecular weight cutoff, $340 \mu \mathrm{m}$ outer diameter with an active surface of 3.0 or $1.0 \mathrm{~mm}$ for the striatal and nigral probes, 
Microdialysis study

levodopa +/-

i. saline

ii. levodopa + saline

iii. levodopa + safinamide
Safinamide

impl

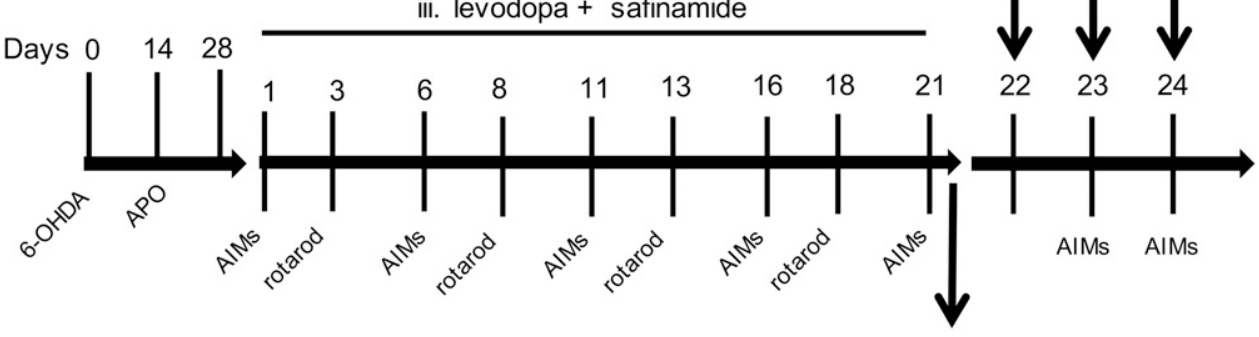

Fig. 1. Schematic representation of the study design.

\section{Molecular study}

PSD-95, GluN2A, GluN2B, GluN2B-pTyr1472, GluA1, GluA1pThr831, GluA1-pThr845, GluA2

respectively). Twenty-four hours after surgery (day 1 session), probes were thoroughly flushed $(3.0 \mu \mathrm{l} / \mathrm{min})$ with a modified Ringer's solution $\left(\mathrm{CaCl}_{2} 1.2 \mathrm{mmol} / \mathrm{l}, \mathrm{KCl} 2.7 \mathrm{mmol} / \mathrm{l}, \mathrm{NaCl} 148 \mathrm{mmol} / \mathrm{l}\right.$, and $\mathrm{MgCl}_{2}$ $0.85 \mathrm{mmol} / \mathrm{l})$. After 6 hours of rinsing, four baseline samples were collected, and then saline or safinamide was administered followed (30 minutes later) by levodopa. Rats chronically treated with levodopa only received the saline/levodopa combination, whereas rats chronically treated with safinamide plus levodopa received the safinamide/levodopa combination.

Saline-treated rats (controls) were implanted according to the procedures described previously. These rats were subjected to the same procedures described for levodopa-treated animals, with the difference being that they did not receive any pharmacological treatment and only four basal samples were collected on day 1 , and when possible on day 2 . Sample collection was every 20 minutes. At the end of the experiment, rats were sacrificed, and brains were excised and processed for verification of probe location.

Glu and GABA were measured by high-performance liquid chromatography coupled with fluorometric detection as previously described (Marti et al., 2008; Mela et al., 2012; Paolone et al., 2015). Thirty microliters of $o$-phthaldialdehyde/mercaptoethanol reagent was added to $30 \mu \mathrm{l}$ aliquots of the sample, and $50 \mu \mathrm{l}$ of the mixture was automatically injected (Triathlon autosampler; Spark Holland, Emmen, Netherlands) onto a 5-C18 Hypersil ODS analytical column (3 $\mathrm{mm}$ inner diameter, $10 \mathrm{~cm}$ length; Thermo Fisher Scientific, Waltham, MA) perfused at a flow rate of $0.48 \mathrm{ml} / \mathrm{min}$ (Jasco PU-2089 Plus quaternary pump; Jasco, Tokyo, Japan) with a mobile phase containing $0.1 \mathrm{M}$ sodium acetate, $10 \%$ methanol, and $2.2 \%$ tetrahydrofuran ( $\mathrm{pH}$ 6.5). Glu and GABA were detected by means of a FP-2020 Plus fluorescence spectrophotometer (Jasco) with the excitation and emission wavelengths set at 370 and $450 \mathrm{~nm}$, respectively. Under these conditions, the limits of detection for Glu and GABA were $\sim 1$ and $\sim 0.5 \mathrm{nM}$, respectively, and their retention times were $\sim 3.5$ and $\sim 18.0$ minutes, respectively.

Synaptic Fraction Purification and Western Blotting. Sixty minutes after the end of chronic treatment (day 21), dyskinetic rats were deeply anesthetized and sacrificed by decapitation. Subcellular fractionation was performed as previously described (Gardoni et al., 2006) with few modifications to the protocol. Briefly, striata were homogenized with a hand-held Teflon-glass homogenizer in ice-cold buffer ( $\mathrm{pH}$ 7.4) containing $0.32 \mathrm{M}$ sucrose, $1 \mathrm{mM}$ Hepes, $1 \mathrm{mM} \mathrm{MgCl}$, $1 \mathrm{mM} \mathrm{NaHCO}$, and 0.1 phenylmethanesulfonyl fluoride in the presence of protease and phosphatase inhibitor cocktails. An aliquot of the homogenate was stored at $-80^{\circ} \mathrm{C}$, while the rest of the sample was centrifuged at $800 \mathrm{~g}$ for 5 minutes to remove nuclear contamination and white matter. The resulting supernatant was spun at $13,000 \mathrm{~g}$ for 15 minutes at $4^{\circ} \mathrm{C}$ to obtain a crude membrane fraction
(P2 fraction) as a pellet, which was resuspended with a glass-glass homogenizer in $1 \mathrm{mM}$ Hepes supplemented with Complete Protease Inhibitor Cocktail Tablets (Roche Diagnostics, Basel, Switzerland). The sample was centrifuged at $100,000 \mathrm{~g}$ for 1 hour at $4^{\circ} \mathrm{C}$. The new pellet was resuspended in $75 \mathrm{mM} \mathrm{KCl}$ and $1 \%$ Triton $\mathrm{X}-100$ and spun at $100,000 \mathrm{~g}$ for 1 hour at $4^{\circ} \mathrm{C}$. The final pellet [Triton X-100insoluble fraction (TIF)] was homogenized in a glass-glass potter in $20 \mathrm{mM}$ Hepes supplemented with Complete Protease Inhibitor Cocktail Tablets (Roche Diagnostics), and then stored at $-80^{\circ} \mathrm{C}$. We isolated the TIF instead of the classic postsynaptic density (PSD) because of the limited amount of starting material. The purity of the TIF fraction was carefully assessed to exclude the presence of presynaptic markers (i.e., Rab3A).

Western blot analysis was performed in homogenate and TIF fractions. All samples were standardized at $1 \mu \mathrm{g} / \mu \mathrm{l}$ concentration. Then, $20 \mu \mathrm{g}$ of each sample was loaded onto an acrylamide/bisacrylamide gel at the appropriate concentration (7\%-9\%), transferred to a nitrocellulose membrane, and probed with primary and horseradish peroxidase-conjugated secondary antibodies. Membrane development was performed using the Chemidoc Imaging System (BioRad, Segrate, Italy) and ImageLab software (BioRad). For quantification, each protein was normalized against the corresponding tubulin band.

Statistical Analysis. All data are expressed as mean \pm S.E.M. Behavioral data are presented in absolute values (AIM scores and time on rod), whereas microdialysis data are presented as percentage of basal levels (calculated as the average of the two samples preceding the treatment). This normalization was adopted in this and previous studies (see Morari et al., 1996; Paolone et al., 2015) to take into account variability in baseline levels across rats and experimental sessions. For neurochemical studies, statistical analysis was performed with two-way analysis of variance (ANOVA) followed by the Bonferroni post-hoc test. Absolute basal neurotransmitter levels in the different rat cohorts were compared using one-way ANOVA followed by the Newman-Keuls post-hoc test. For molecular studies, statistical analysis was performed with one-way ANOVA followed by Tukey's post-hoc test.

Materials. Safinamide methansulfonate (batch FN1291501) was provided by Zambon SpA (Bresso, Italy), dissolved in saline, and administered intraperitoneally as free base. 6-OHDA hydrobromide, dissolved in $0.02 \%$ ascorbate-saline, was purchased from Tocris (Bristol, United Kingdom). Levodopa, benserazide hydrochloride, and apomorphine hydrochloride were purchased from Sigma-Aldrich (Milan, Italy). Clarity Western ECL Substrate was purchased from BioRad. Complete Protease Inhibitor Cocktail Tablets and phosSTOP Phosphatase Inhibitor Cocktail Tablets were purchased from Roche Diagnostics. For western blot analysis, the following unconjugated primary antibodies were used: polyclonal anti-GluN2A 

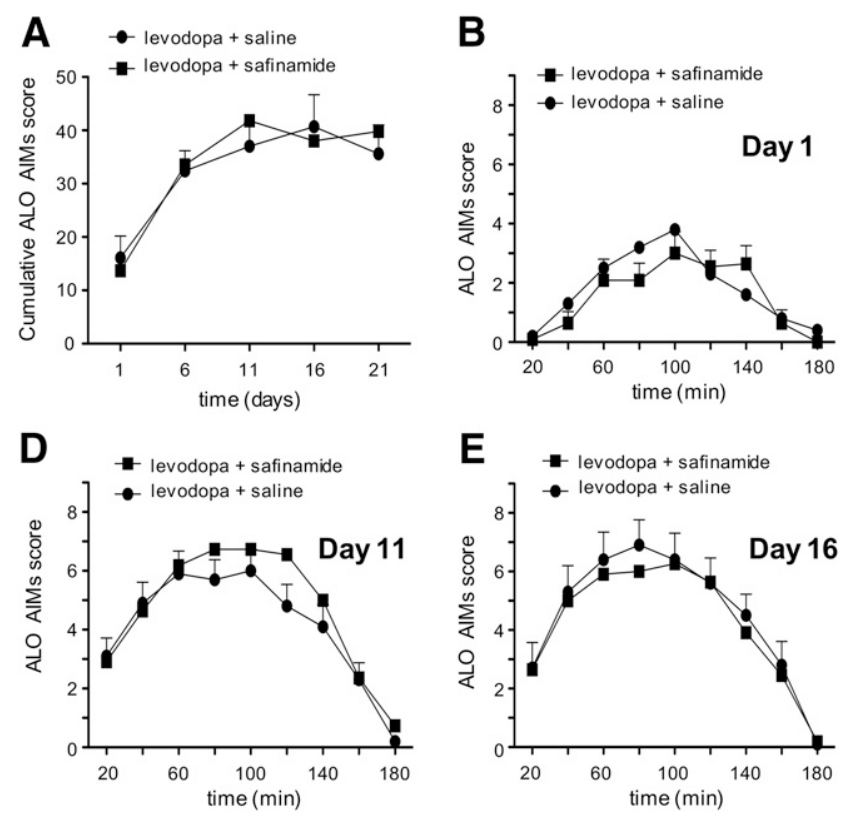

antibody (1:1000; Sigma-Aldrich); monoclonal anti-GluN2B antibody (1:2000; NeuroMab, Davis, CA); polyclonal anti-phospho-GluN2B (Tyr1472, 1:1000; Cell signaling, Leiden, The Netherlands); monoclonal anti-PSD-95 (1:3000; NeuroMab); polyclonal anti-GluA1 antibody (1:2000; Merck Millipore, Milan, Italy); monoclonal anti-GluA2 antibody (1:1000; NeuroMab); polyclonal anti-phospho-GluA1 (Ser831, 1: 1000; Merck Millipore); polyclonal anti-phospho-GluA1 (Ser845, 1: 1000; Merck Millipore); polyclonal anti-Rab3A (1:2000; Abcam, Cambridge, United Kingdom); monoclonal anti- $\alpha$-tubulin antibody (1: 10,000; Sigma-Aldrich); and polyclonal antityrosine hydroxylase (1: 3000; Merck Millipore).

\section{Results}

Lack of Effect of Safinamide on LID Development. Levodopa (6 mg/kg, plus benserazide, $12 \mathrm{mg} / \mathrm{kg}$ ) alone or in association with safinamide ( $15 \mathrm{mg} / \mathrm{kg}$ i.p., 30 minutes before levodopa) was administered for 3 weeks to 6-OHDA parkinsonian rats (see Fig. 1). As previously demonstrated (Cenci et al., 2007; Gardoni et al., 2012; Marti et al., 2012; Paolone et al., 2015), parkinsonian rats chronically treated with levodopa develop AIMs (Fig. 2A). Dyskinetic movements in 6-OHDA hemilesioned rats treated with saline plus levodopa $(n=10)$ appeared after the first levodopa injection (ALO AIM scores of $16.1 \pm 4.1$ ) and progressively increased during levodopa treatment, reaching a maximal value of $40.7 \pm 6.0$ at day 16 (Fig. 2A). The time course of AIM responses after acute levodopa injection was qualitatively similar from day 1 to 21 (Fig. 2, B-F): ALO AIMs developed gradually, reached maximal values between 60 and 100 minutes, and then gradually declined and disappeared 3 hours after levodopa administration.

The time course of AIM development was similar in 6-OHDA hemilesioned rats treated with levodopa in combination with safinamide (safinamide/levodopa, $n=11$ ) or with saline (saline/levodopa, $n=10$; Fig. 2A). Safinamide-treated rats became stably dyskinetic after a week of treatment (33.5 \pm 5.4 after 6 days) and showed a maximal ALO AIM score of $41.8 \pm 5.1$ at day 11. Likewise, the acute response to levodopa of safinamide/levodopa-treated rats throughout the
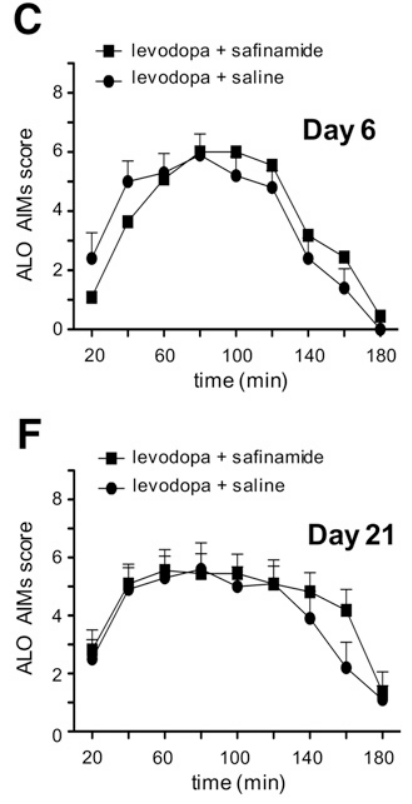

Fig. 2. Chronic safinamide did not modify the development of levodopa-induced dyskinesia in 6-OHDA hemilesioned rats. Rats were treated once daily for 21 days with levodopa $(6 \mathrm{mg} / \mathrm{kg}$ + benserazide $12 \mathrm{mg} / \mathrm{kg}$, s.c.) in combination with saline or safinamide ( $15 \mathrm{mg} / \mathrm{kg}$; i.p.), given 30 minutes prior to levodopa. ALO AIMs were scored on day $1(\mathrm{~B})$, day $6(\mathrm{C})$, day $11(\mathrm{D})$, day $16(\mathrm{E})$ and day $21(\mathrm{~F})$, according to a severity (frequency) scale. Cumulative ALO AIM scores is shown (A). Data are expressed as mean \pm S.E.M. of 10 (levodopa + saline group) or 11 (levodopa + safinamide group) rats.

treatment period (Fig. 2, B-F) was superimposable to that of saline/levodopa-treated rats. Locomotive AIMs (i.e., contralateral turning behavior) also showed a gradual increase over levodopa treatment (Fig. 3); however, again, no significant difference between groups was observed.

To investigate whether safinamide could influence rat motor ability along with chronic levodopa treatment, rotarod performance was evaluated pre- and post-levodopa (Fig. 4). Saline/levodopa- or safinamide/levodopa-treated rats showed similar rotarod performances before levodopa injection (off levodopa; time on rod: $601 \pm 28$ and $595 \pm 32$ seconds, respectively, at day 3). Rotarod performance monitored $60 \mathrm{~min}$ utes after levodopa administration (on levodopa) at day 3 was equally reduced by about $25 \%$ in both treatment groups. Further testing sessions along with levodopa treatment revealed that basal rotarod performance (before levodopa treatment) remained stable in both groups, although as expected the degree of motor impairment after levodopa was enhanced, likely due to development of more severe AIMs.

Effect of Safinamide on Neurochemical Markers of LID. Twenty-four hours after the end of chronic treatments (i.e., at day 22), saline/levodopa- and safinamide/levodopa-treated rats underwent microdialysis probe implantation in the DA-depleted dorsolateral striatum and ipsilateral SNr. Twenty-four hours after surgery rats were administered the same treatments they received during the chronic study, and AIMs were monitored for 2 hours simultaneously with sample collection.

In rats subjected to microdialysis, dyskinetic movements appeared soon after levodopa administration (Fig. 5A). The time course of AIM response was similar in saline/levodopaand safinamide/levodopa-treated rats, and the ALO AIM scores during microdialysis were similar in both groups (23.9 \pm 3.2 and $23.4 \pm 2.9, n=15$ and $n=13$, respectively). These responses were also similar to those observed during the last testing session (day 21), i.e., before microdialysis surgery, suggesting probe implantation did not affect the behavioral response to levodopa in the two groups.

Since it was proven that the rise in SNr GABA during LID reflects the activation of the direct striato-nigral pathway 
A

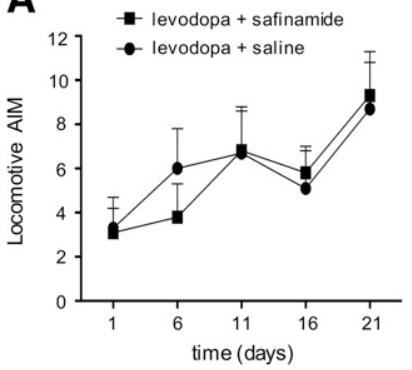

D

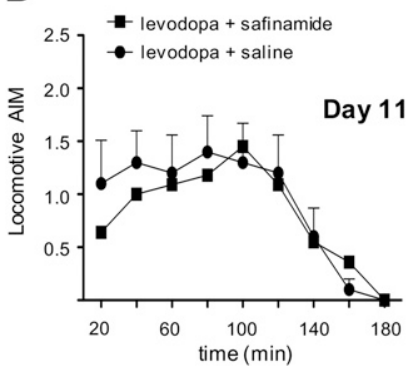

B

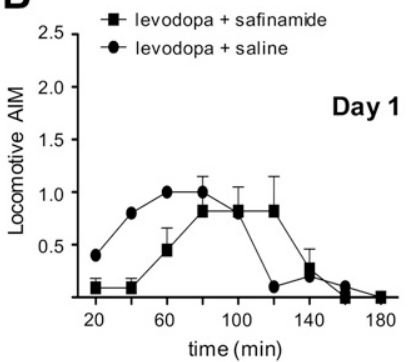

E

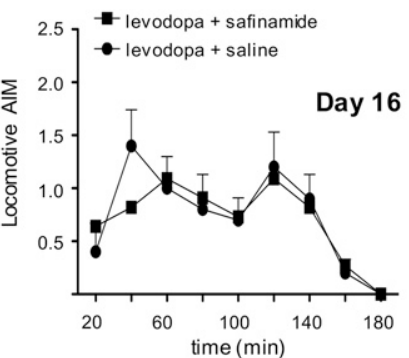

C

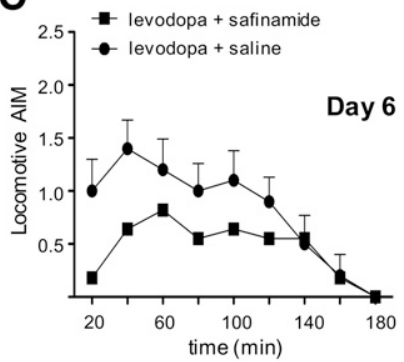

$\mathbf{F}$

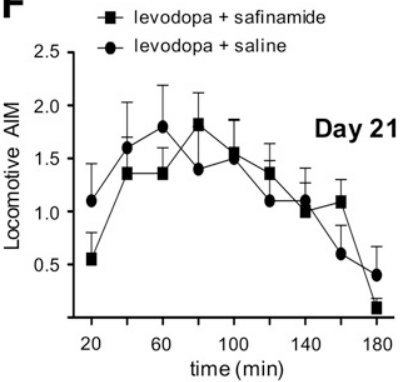

Fig. 3. Chronic safinamide did not modify the development of locomotive dyskinesia (i.e., contralateral turning behavior) in 6-OHDA hemilesioned rats. Rats were treated once daily for 21 days with levodopa $(6 \mathrm{mg} / \mathrm{kg}$ + benserazide $12 \mathrm{mg} / \mathrm{kg}$, s.c.) in combination with saline or safinamide $(15 \mathrm{mg} / \mathrm{kg}$, i.p.), given 30 minutes prior to levodopa. Locomotive AIMs were scored on day 1 (B), day 6 (C), day 11 (D), day $16(\mathrm{E})$ and day $21(\mathrm{~F})$, according to a severity (frequency) scale for 3 hours following levodopa administration. $\mathrm{Cu}$ mulative AIM scores are shown (A). Data are expressed as mean \pm S.E.M. of 10 (levodopa + saline group) or 11 (levodopa + safinamide group) rats
(Mela et al., 2007, 2012; Marti et al., 2012), the ability of safinamide to modulate nigral GABA was assessed (Fig. 5B). In $\mathrm{SNr}$, the basal GABA levels (Table 1) were $3.67 \pm 0.41 \mathrm{nM}$ in the saline/levodopa group $(n=15)$ and $3.18 \pm 0.56 \mathrm{nM}$ in the safinamide/levodopa group $(n=13)$. Of note, the GABA levels in the safinamide/levodopa group were significantly lower $(P<0.05)$ than the SNr GABA levels in 6-OHDA hemilesioned, levodopa unprimed rats $(5.39 \pm 0.86 n=10)$ but not different from the GABA levels in the saline/levodopa group (Fig. 5B) $\left(F_{2,35}=3.36, P<0.05\right)$. Acute levodopa challenge in saline/levodopa-treated rats caused marked elevation of nigral GABA levels that reached a maximum of $140 \%$ over baseline at 80 and 100 minutes after levodopa administration (Fig. 5B). The response of nigral GABA to levodopa in safinamide/levodopa-treated rats was superimposable to that of saline/levodopa-treated rats (treatment: $F_{1,9}=0.25, P=$ 0.62 ; time: $F_{9,234}=12.23, P<0.0001$; time $\times$ treatment interaction: $F_{9,234}=0.32, P=0.98$ ) (Fig. $5 \mathrm{~B}$ ).

As a result, the ability of safinamide to affect the rise in striatal Glu associated with LID appearance was investigated. Basal Glu levels (Table 1) in striatum were not different in saline/levodopa- and safinamide/levodopa-treated rats $(117.01 \pm 16.64$ and $133.43 \pm 22.68 \mathrm{nM}$, respectively; $n=$ 15 and $n=13$, respectively), and in turn these values were not different from those found in saline-treated 6-OHDA hemilesioned rats (Fig. 5C; $123.87 \pm$ 19.74). Statistical analysis revealed a significant inhibitory effect of safinamide on levodopa-induced striatal Glu release (Fig. 5C; treatment: $F_{1,9}=9.53, P=0.0047$; time: $F_{9,234}=2.34, P=0.0154$ : time $\times$ treatment interaction: $\left.F_{9,234}=2.76, P=0.0043\right)$. Safinamide
A

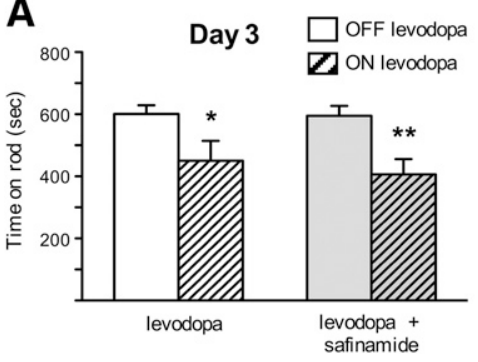

C

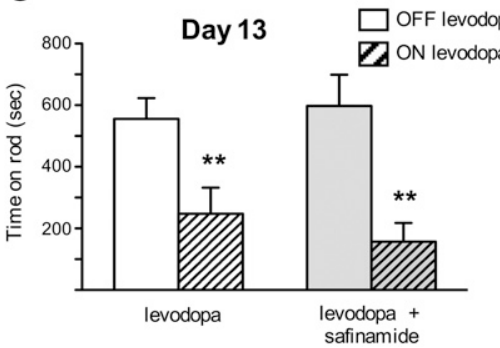

B

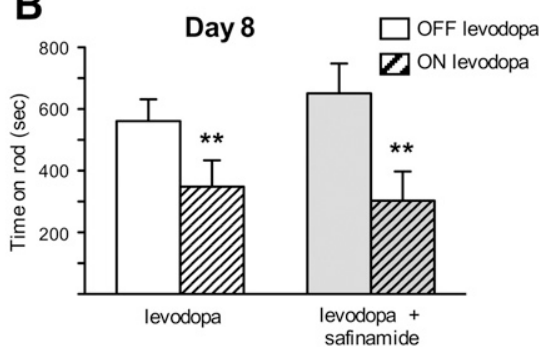

D

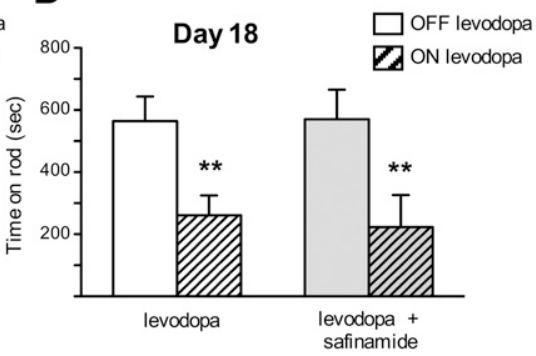

Fig. 4. Chronic safinamide failed to affect the impairment of rotarod performance in dyskinetic rats after levodopa administration. Rats were treated once daily for 21 days with levodopa $(6 \mathrm{mg} / \mathrm{kg}+$ benserazide $12 \mathrm{mg} / \mathrm{kg}$, s.c. $)$ in combination with saline or safinamide $(15 \mathrm{mg} / \mathrm{kg}$, i.p.), given 30 minutes prior to levodopa. Rotarod performance (time on rod in seconds) was measured on day 3 (A), day 8 (B), day 13 (C), and day 18 (D), both before (off levodopa) and 60 minutes after levodopa (on levodopa) administration. Data are expressed as mean \pm S.E.M. of 10 (levodopa + saline group) or 11 (levodopa + safinamide group) rats. Statistical analysis was performed by the Student's $t$ test, two-tailed for paired data. ${ }^{*} P<0.05 ;{ }^{*} P<0.01$, different from before levodopa treatment. 

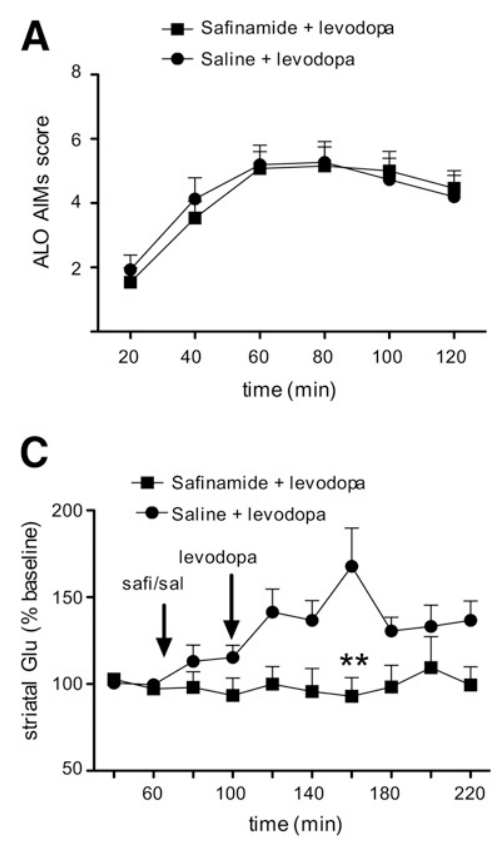
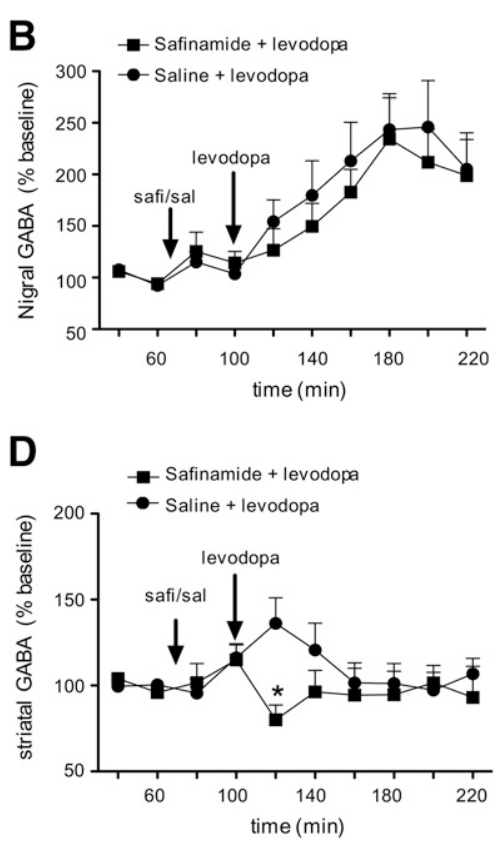

Fig. 5. Safinamide prevented the rise in striatal GABA and glutamate levels induced by levodopa challenge in dyskinetic rats subjected to microdialysis. Rats were treated once daily for 21 days with levodopa $(6 \mathrm{mg} / \mathrm{kg}+$ benserazide $12 \mathrm{mg} / \mathrm{kg}$, s.c.) in combination with saline or safinamide $(15 \mathrm{mg} / \mathrm{kg}$, i.p.), given 30 minutes prior to levodopa. At the end of treatment, rats were implanted with a microdialysis probe in the DA-depleted striatum and another in the ipsilateral SNr. Twenty-four hours later, rats were administered a challenge dose of levodopa in combination with saline or safinamide $15 \mathrm{mg} / \mathrm{kg}$ (given 30 minutes prior to levodopa). (A) Safinamide did not modify the expression of levodopa-induced dyskinesia in dyskinetic rats subjected to microdialysis. (B) Effect of safinamide on nigral GABA. (C) Effect of safinamide on striatal glutamate. (D) Effect of safinamide on striatal GABA. Data are expressed as percentage of prestimulation levels (calculated as the mean between the two samples preceding the treatment) and are the mean \pm S.E.M. values of 15 (levodopa + saline group) or 13 (levodopa + safinamide group) determinations. Statistical analysis was performed by two-way ANOVA followed by the Bonferroni post-hoc test. ${ }^{*} P<0.05 ; * * P<0.01$ different from saline + levodopa. inhibition was significant $(P<0.01)$ at the 160 -minute time point.

The ability of chronic safinamide of modulating striatal circuitry was also evident during monitoring of striatal GABA release (Fig. 5D). Basal GABA levels in striatum were similar in both saline/levodopa- and safinamide/levodopa-treated rats $(4.61 \pm 0.90$ and $5.10 \pm 1.11 \mathrm{nM}$, respectively; $n=15$ and $n=$ 13 , respectively). These values were not different from those observed in 6-OHDA hemilesioned, levodopa unprimed rats chronically treated with saline (Fig. 5D; $5.92 \pm 1.11 ; n=10$ ). Two-way ANOVA revealed that safinamide prevented the rise in striatal GABA levels induced by levodopa (Fig. 5D) [nonsignificant effect of treatment $\left(F_{1,9}=1.35, P=0.25\right)$ or time $\left(F_{9,234}=0.0 .80, P=0.0 .61\right)$, but a time $\times$ treatment interaction was statistically significant $\left.\left(F_{9,234}=1.92, P=0.05\right)\right]$. The effect of safinamide was significant at the 120-minute time point $(P<0.05)$.

Effect of Safinamide on Molecular Markers of LID at the Excitatory Synapse. To understand the molecular mechanisms at the glutamatergic synapse underlying the effect of safinamide, a postsynaptic TIF was purified from the contralateral and ipsilateral striata of the 6-OHDA lesioned rats. This fraction was previously used to characterize the alterations in the PSD compartment in experimental models of PD and LID and represents an ideal experimental tool for the evaluation of synaptic markers of dyskinesia at the striatal glutamatergic synapse (Gardoni et al., 2006, 2012; Mellone et al., 2015; Stanic et al., 2017).

Among the different adaptive changes taking place in the striatum after chronic levodopa treatment, alterations in the trafficking and localization of NMDAR regulatory subunits at the postsynaptic membrane have been shown (Mellone and Gardoni, 2018). In particular, the onset of LID is associated with the rearrangement of NMDAR GluN2-type subunits, leading to an increase in the GluN2A/GluN2B subunit ratio in the postsynaptic compartment of SPNs (Gardoni et al., 2012; Mellone et al., 2015; Stanic et al., 2017). In agreement with these previous studies, a significant increase in GluN2A levels in striatal postsynaptic membranes of dyskinetic levodopatreated rats compared with 6-OHDA lesioned rats was found (Fig. 6, A and C; $F_{5,42}=4.073, P=0.0042$, one-way ANOVA, Tukey's post-hoc comparison; GluN2A, ipsilateral levodopa vs. ipsilateral, $n=8, * P<0.05)$, and it was paralleled by a concomitant increase in the synaptic GluN2A/GluN2B ratio (Fig. 6, A and B; $F_{5,42}=3.436, P=0.0108$, one-way ANOVA, Tukey's post-hoc comparison; GluN2A/GluN2B, ipsilateral levodopa vs. ipsilateral, $n=8, * P<0.05)$. Notably, concomitant treatment with safinamide was able to completely reverse the previously described alterations of GluN2A and GluN2A/2B synaptic levels induced by levodopa (Fig. 6, A-C;

TABLE 1

Basal dialysate levels (mean \pm S.E.M.) monitored in the different experimental conditions/treatment groups

\begin{tabular}{lccc}
\hline & & \multicolumn{2}{c}{ Striatum } \\
\cline { 3 - 4 } Treatment in 6-OHDA Lesioned Rats & SNr GABA & GABA & Glutamate \\
\hline & $n M$ & $n M$ & $n M$ \\
Saline/L-DOPA $(n=15)$ & $3.67 \pm 0.41$ & $4.61 \pm 0.90$ & $117.01 \pm 16.64$ \\
Safinamide/L-DOPA $(n=13)$ & $3.18 \pm 0.56^{*}$ & $5.10 \pm 1.11$ & $133.43 \pm 22.68$ \\
Saline $(n=10)$ & $5.39 \pm 0.86$ & $5.92 \pm 0.11$ & $123.87 \pm 19.74$ \\
\hline$* P<0.05$ from 6-OHDA saline. & & &
\end{tabular}

$* P<0.05$ from 6-OHDA saline. 

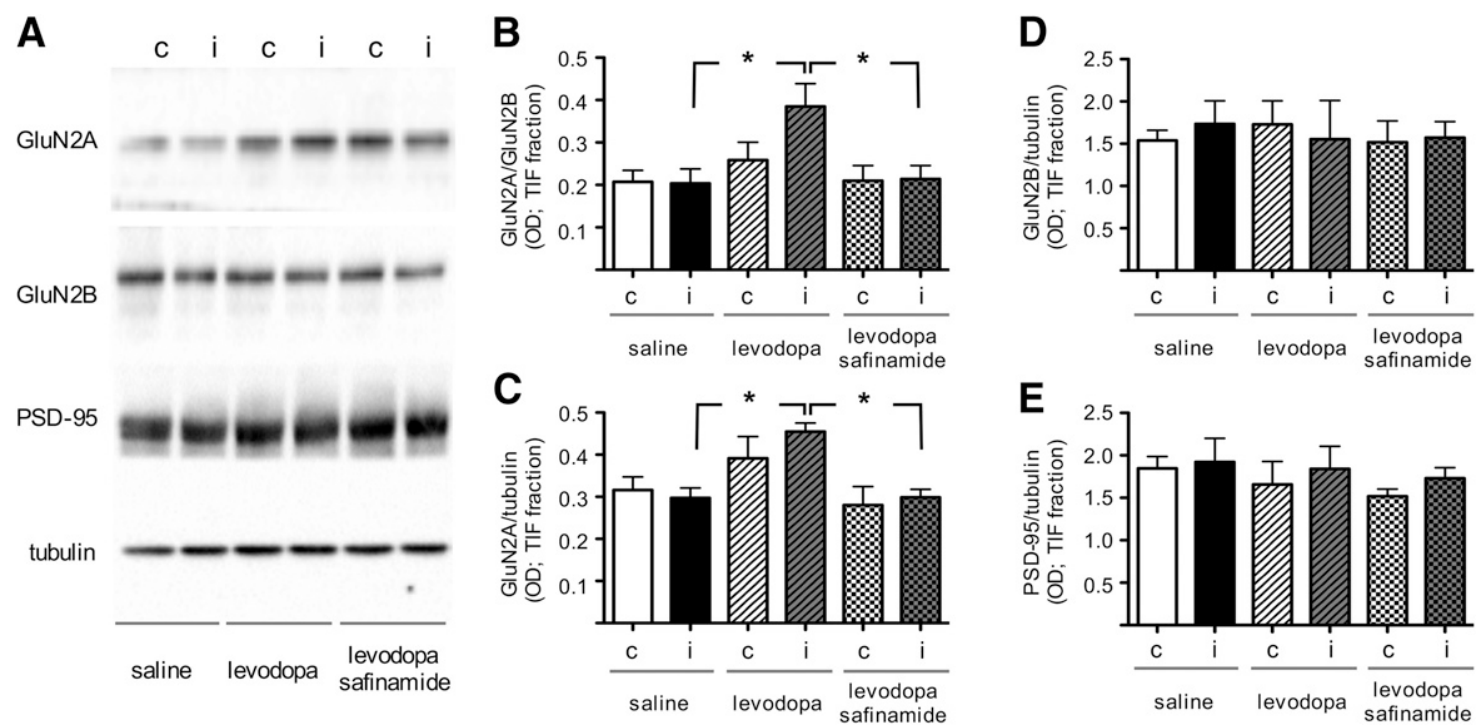

Fig. 6. (A-E) Molecular analysis of the corticostriatal synapse in parkinsonian animals treated with levodopa plus safinamide. Western blot analysis was performed on the postsynaptic TIF fraction for glutamate receptor subunits GluN2A and GluN2B and the scaffolding protein PSD-95. Protein levels are expressed as optical density (OD) following normalization on tubulin levels (B-E). For each protein, representative blots are shown (A). Data are presented as mean \pm S.E.M., statistical analysis was performed with one-way ANOVA followed by Tukey's post-hoc test $(n=8)$, ${ }^{*} P<0.05$; "c" denotes contralateral striatum to the 6-OHDA lesion; "i" denotes ipsilateral striatum to the 6-OHDA lesion.

GluN2A, ipsilateral levodopa safinamide vs. ipsilateral levodopa, $n=8,{ }^{*} P<0.05$; GluN2A/GluN2B, ipsilateral levodopa safinamide vs. ipsilateral levodopa, $n=8,{ }^{*} P<0.05$ ). In agreement with previous reports, there were no significant changes in the synaptic localization of the GluN2B subunit (Fig. 6, A and D; GluN2B, $F_{5,42}=0.1211, P=0.9870$, one-way ANOVA, Tukey's post-hoc comparison). Finally, no alterations of synaptic levels of PSD-95, the most abundant scaffolding protein of the excitatory PSD, were found (Fig. 6, A and E; PSD-95, $F_{5,42}=0.4973, P=0.7764$, oneway ANOVA, Tukey's post-hoc comparison).

Previous conflicting studies suggested a possible involvement of GluN2B tyrosine phosphorylation (GluN2B pTyr1472) in PD as well as in LIDs (Menegoz et al., 1995; Dunah et al., 2004; Napolitano et al., 2006; Ba et al., 2014). As previously reported by our group in a 6-OHDA rat model (Gardoni et al., 2006), no significant difference in the ratio of pTyr1472-GluN2B/total GluN2B following chronic treatment with levodopa was observed (Fig. 7, A and B; pTyr1472-GluN2B/total GluN2B, $F_{5,42}=0.2318, P=0.9465$, one-way ANOVA, Tukey's post-hoc comparison; ipsilateral levodopa vs. ipsilateral, $n=8, P>$ 0.05); similarly, no modification of pTyr1472-GluN2B was induced by concomitant treatment with safinamide (Fig. 7, $A$ and $B$; ipsilateral levodopa safinamide vs. ipsilateral levodopa, $n=8, P>0.05$ ).
Alterations in AMPAR phosphorylation at synapses represent an additional validated marker of LID in animal models (Santini et al., 2007; Silverdale et al., 2010; Ghiglieri et al., 2016). In particular, increased PKA-dependent phosphorylation of GluA1-pSer845 (Roche et al., 1996; Barria et al., 1997), which is correlated with the activation of the D1/PKA/Darpp32 signaling pathway (Picconi et al., 2003; Aubert et al., 2005; Santini et al., 2010) and the surface retention of AMPARs, has been described in rodent (Santini et al., 2007; Errico et al., 2011; Ghiglieri et al., 2016) and macaque (Santini et al., 2010) models of LID. The levels of GluA1-pSer845 in the postsynaptic compartment purified from contralateral and ipsilateral striata were measured. As expected, a significant increase of PKA-related activity in the GluA1p845 site was found in the ipsilateral striatum of levodopa-treated dyskinetic animals compared with the lesioned one (Fig. 8, A and B; GluA1-pSer845, $F_{5,42}=2.999, P=0.0210$, one-way ANOVA, Tukey's post-hoc comparison; ipsilateral levodopa vs. ipsilateral, $\left.n=8,{ }^{*} P<0.05\right)$. No alterations of GluA1 pSer831 phosphorylation by protein kinase $\mathrm{C}$ and CaMKII (Roche et al., 1996; Barria et al., 1997) were observed in any experimental group (Fig. 8, A and B; GluA1-pSer831, $F_{5,42}=2373, P=0.9438$, one-way ANOVA, Tukey's post-hoc comparison). Treatment with safinamide failed to reduce the levels of pGluA1-pSer845
A

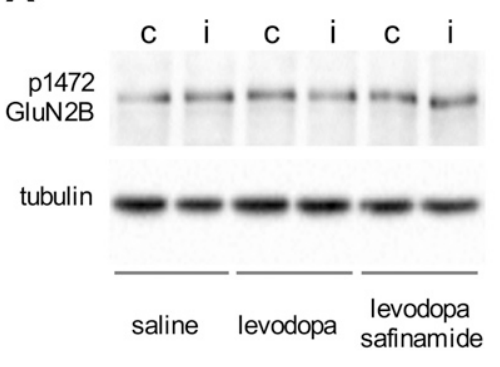

B

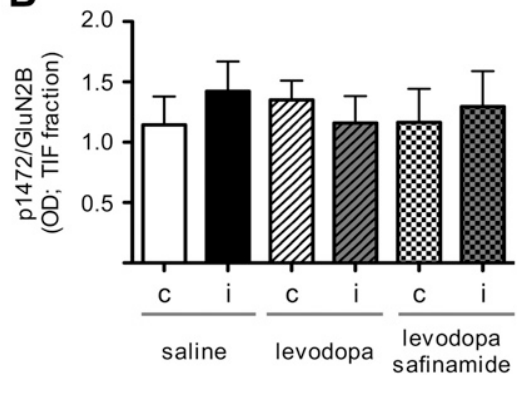

Fig. 7. (A and B) Molecular analysis of the corticostriatal synapse in parkinsonian animals treated with levodopa plus safinamide. Western blot (WB) analysis was performed on the postsynaptic TIF fraction for glutamate receptor subunits GluN2BpTyr1472 and tubulin. Protein levels are expressed as optical density (OD) following normalization on tubulin and total GluN2B levels (B; for representative WB see Fig. 6). Representative blots for GluN2B p1472 and tubulin are shown (A). Western blotting was repeated three times for all samples. Data are presented as mean \pm S.E. M., and statistical analysis was performed with one-way ANOVA followed by Tukey's post-hoc test $(n=8)$; "c" denotes contralateral striatum to the 6-OHDA lesion; "i" denotes ipsilateral striatum to the 6-OHDA lesion. 
A

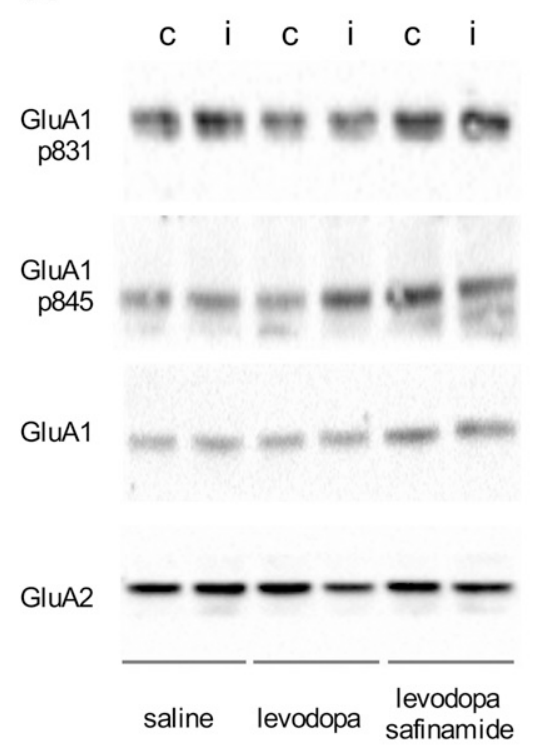

B

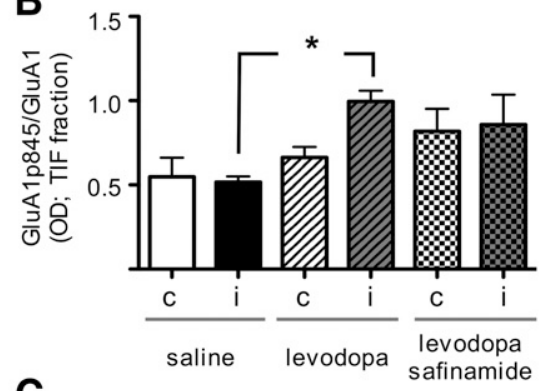

C

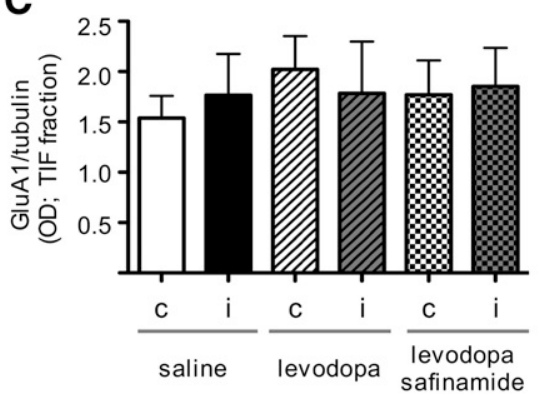

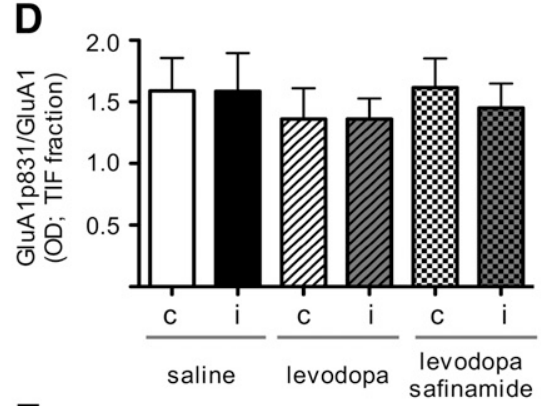

E

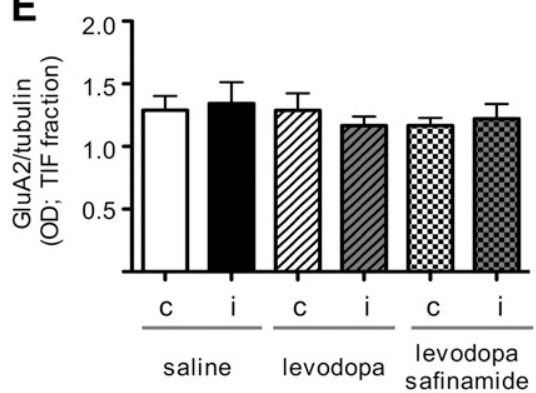

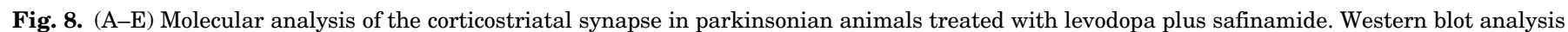

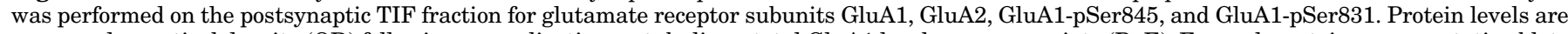

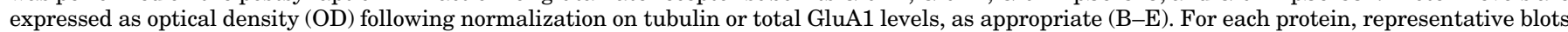

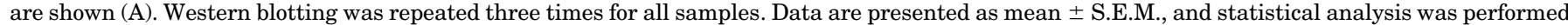

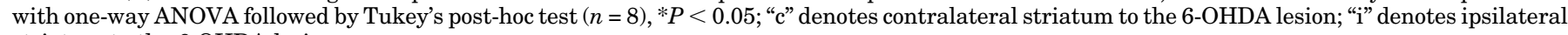
striatum to the 6-OHDA lesion.

(Fig. 8, A and B; GluA1-pSer845, ipsilateral levodopa safinamide vs. ipsilateral levodopa, $n=8, P>0.05$ ). However, pGluA1-pSer845 levels in the safinamide-treated group did not differ from the saline-treated parkinsonian animals (Fig. 8, A and B; GluA1-pSer845, ipsilateral levodopa safinamide vs. ipsilateral, $n=8, P>0.05)$. Finally, the total synaptic amount of AMPAR subunits (GluA1 and GluA2) was not altered by the different treatments (Fig. 8, A, D, and E; GluA1, $F_{5,42}=0.2008, P=0.9715$; GluA2, $F_{5,42}=0.04307, P=$ 0.8609; one-way ANOVA, Tukey's post-hoc comparison).

\section{Discussion}

In a previous microdialysis study (Morari et al., 2018) we showed that a dose of safinamide as high as $15 \mathrm{mg} / \mathrm{kg}$ was able to inhibit the veratridine-evoked Glu release in the subthalamic nucleus, globus pallidus, and $\mathrm{SNr}$ of naive rats. Of note, this dose generated free brain concentrations of safinamide that were slightly less than $2 \mu \mathrm{M}$, which is close to the $K_{\mathrm{i}}$ value of safinamide at voltage-operated sodium channels and to the estimated free brain concentrations reached in patients at therapeutic doses.

We now report that chronic administration of $15 \mathrm{mg} / \mathrm{kg}$ safinamide to 6-OHDA lesioned rats did not modify the time course of LID development or the levodopa-induced motor impairment associated with acute dyskinesia appearance. Nonetheless, the same dose of safinamide induced significant inhibition of striatal well-validated biochemical and neurochemical correlates of dyskinesia, namely, the striatal rearrangement of synaptic GluN2 subunits of the NMDAR and the levodopa-induced rise of striatal Glu release.

The failure of safinamide in inhibiting LID expression in rats is in sharp contrast to the antidyskinetic effect of acute and subacute safinamide $(3-30 \mathrm{mg} / \mathrm{kg})$ in female macaques
(Grégoire et al., 2013). The discrepancy might be a result of differences in the models and the experimental protocols adopted in the two studies. This may be due to a different target representation in the two species. For instance, it is known that MAO-B plays a relevant role in brain DA metabolism in the primate but not in the rat (Glover et al., 1980; Youdim et al., 2006). Moreover, voltage-operated sodium channels can present with different physio/pharmacological properties in the primate and in the rat brains (Zhang et al., 2017).

The failure of safinamide to inhibit LID development and expression in the rat is, however, consistent with the failure to inhibit the accompanying rise in nigral GABA. This response is a neurochemical marker of striato-nigral direct pathway activation, and relies on striatal D1 receptor stimulation (Mela et al., 2012). Therefore, the fact that safinamide is not able to modulate this pathway in the rat model of dyskinesia, at least in the time frame considered, i.e., the classic 3 week levodopa administration protocol (Cenci et al., 1998), confirms that safinamide does not bind to the D1 receptor (Salvati et al., 1999) and suggests that it does not enhance dopaminergic tone. In fact, in the rat, DA is deaminated mainly by monoamine oxidase A, with MAO-B playing a minor role (SaderMazbar et al., 2013).

Despite being ineffective on LID, safinamide prevented the rise in striatal Glu associated with dyskinesia appearance. Although no study has thoroughly investigated the neurobiological substrates of this neurochemical response, the rise of striatal Glu is thought to reflect the increase in Glu release from cortico-striatal terminals associated with motor activation (Sgambato-Faure and Cenci, 2012). Pyramidal glutamatergic neurons project to the striatum and are the driving force of striatofugal SPNs. In support of this theory, altered synaptic plasticity-namely, loss of synaptic depotentiation-has been observed at cortico-striatal terminals (Picconi et al., 2003). 
Inhibition of pyramidal neurons (with a 5-HT1A agonist) consistently attenuated both the rise of striatal Glu and the expression of LID (Ostock et al., 2011). Moreover, the mixed 5-HT1A/5-HT1B receptor agonist eltoprazine prevented both LID expression and nigral and striatal amino acid release (Paolone et al., 2015). We should, however, recall that other microdialysis studies have reported a dissociation between dyskinesia appearance and striatal Glu dynamics (Dupre et al., 2011, Mela et al., 2012; Brugnoli et al., 2016), questioning the view that the increase in striatal dialysate Glu is a marker of LID and eventually instrumental in the expression of dyskinesia.

As a possible behavioral counterpart of the striatal antiglutamatergic action of safinamide, we should mention a study reporting that reduction of striatal glutamatergic transmission correlates with motor improvement (antiakinetic effect) rather than dyskinesia (Gubellini et al., 2006). Highfrequency stimulation of the subthalamic nucleus did not affect LID but ameliorated akinesia in 6-OHDA hemilesioned rats (Gubellini et al., 2006). The electrophysiological ex vivo correlate was that in high-frequency stimulation-treated dyskinetic rats the hyperactivity of striatal SPNs (induced by 6-OHDA and potentiated by levodopa) was dramatically reduced (Gubellini et al., 2006). Microdialysis data would suggest that chronic safinamide alters striatal glutamatergic transmission via the thalamo-cortico-striatal loop. In fact, acute safinamide is capable of inhibiting Glu release in subthalamic nucleus and projection areas (globus pallidus and $\mathrm{SNr}$ ) in naive animals (Morari et al., 2018).

From a molecular point of view, we show here that chronic treatment with safinamide is able to only partially normalize the molecular alteration of the corticostriatal glutamatergic synapse associated with chronic treatment with levodopa (Mellone et al., 2015; Ghiglieri et al., 2016; Mellone and Gardoni, 2018). In particular, previous studies demonstrated that the onset of LID is associated with an alteration of NMDAR GluN2-type subunits, namely an increase in the GluN2A/GluN2B subunit ratio at striatal excitatory synapses (Gardoni et al., 2006, 2012; Mellone et al., 2015), in the absence of any significant alteration of the expression levels of any NMDAR subunit (Calon et al., 2002; Gardoni et al., 2006; Morin et al., 2014; Mellone et al., 2015). Notably, several reports (Bagetta et al., 2012; Tronci et al., 2014; Ghiglieri et al., 2016; Stanic et al., 2017) clearly addressed that pharmacological interventions able to reduce the severity of LID also induced a reverse of the physiologic synaptic GluN2A/GluN2B ratio in the striatum. Here, we observed that safinamide is able to prevent the aberrant increase of the synaptic levels of the GluN2A subunit associated with chronic levodopa treatment. Accordingly, our data suggest that the normalization of striatal Glu release induced by safinamide is also sufficient to prevent the adaptive mechanisms at postsynaptic NMDAR levels. Conversely, safinamide failed to prevent the increased PKA-dependent phosphorylation of GluA1-pSer845 associated with chronic treatment with levodopa. Taking into account that aberrant activation of PKA in the postsynaptic compartment of striatal SPNs mainly relies on striatal D1 receptor overactivation (Mela et al., 2012), biochemical data corroborate microdialysis data endorsing the view that safinamide is not able to modulate the direct pathway activation in this rat model of LID. Moreover, the results presented here suggest that a complete rescue of the molecular alterations of the excitatory synapse, both at NMDAR and AMPAR levels, are needed to observe an antidyskinetic effect of a drug. However, the absence of experimental analysis pointing to involvement of the safinamide effect in other key striatal neurotransmitter systems such as dopamine and acetylcholine might be a limitation of the present study, and further investigations should be addressed.

In conclusion, safinamide did not halt or delay the priming to levodopa, which underlies the development of dyskinesia, in the rat. In addition, safinamide did not affect levodopainduced D1 receptor-mediated direct pathway activation. At the same time, safinamide did not worsen dyskinesia severity, as might be expected from drugs that potentiate levodopa action (i.e., MAO-B inhibitors). Consistent with its antiglutamatergic activity, safinamide inhibited different parameters of corticostriatal glutamatergic transmission, an effect that might correlate with its antiakinetic activity. Overall, the present findings suggest that the striatal Glu-modulating component of safinamide's activity may contribute to its clinical effects of improving motor function and increasing "on" time without troublesome dyskinesia (Borgohain et al., 2014; Schapira et al., 2017).

\section{Acknowledgments}

We thank Elisa Zianni and Annalisa Longhi for excellent technical support.

\section{Authorship Contributions}

Participated in research design: Gardoni, Morari, Kulisevsky, Caccia, Melloni, Padoani, Vailati, Keywood.

Conducted experiments: Brugnoli, Novello, Pisanò, Mellone, Sosti. Performed data analysis: Gardoni, Morari, Brugnoli, Novello, Pisanò, Mellone, Sosti.

Wrote or contributed to the writing of the manuscript: Gardoni, Morari, Caccia.

\section{References}

Aubert I, Guigoni C, Håkansson K, Li Q, Dovero S, Barthe N, Bioulac BH, Gross CE, Fisone G, Bloch B, et al. (2005) Increased $\mathrm{D}_{1}$ dopamine receptor signaling in levodopa-induced dyskinesia. Ann Neurol 57:17-26.

Ba M, Kong M, and Ma G (2014) Postsynaptic density protein 95-regulated NR2B tyrosine phosphorylation and interactions of Fyn with NR2B in levodopa-induced dyskinesia rat models. Drug Des Devel Ther 9:199-206.

Bagetta V, Sgobio C, Pendolino V, Del Papa G, Tozzi A, Ghiglieri V, Giampà C, Zianni E, Gardoni F, Calabresi P, et al. (2012) Rebalance of striatal NMDA/AMPA receptor ratio underlies the reduced emergence of dyskinesia during D2-like dopamine agonist treatment in experimental Parkinson's disease. J Neurosci 32: 17921-17931.

Barria A, Derkach V, and Soderling T (1997) Identification of the $\mathrm{Ca}^{2+} /$ calmodulindependent protein kinase II regulatory phosphorylation site in the $\alpha$-amino-3hydroxyl-5-methyl-4-isoxazole-propionate-type glutamate receptor. $\mathrm{J}$ Biol Chem 272:32727-32730.

Bastide MF, Meissner WG, Picconi B, Fasano S, Fernagut PO, Feyder M, Francardo V, Alcacer C, Ding Y, Brambilla R, et al. (2015) Pathophysiology of L-dopa-induced motor and non-motor complications in Parkinson's disease. Prog Neurobiol 132: 96-168.

Bido S, Marti M, and Morari M (2011) Amantadine attenuates levodopa-induced dyskinesia in mice and rats preventing the accompanying rise in nigral GABA levels. J Neurochem 118:1043-1055.

Bido S, Solari N, Indrigo M, D’Antoni A, Brambilla R, Morari M, and Fasano S (2015) Differential involvement of Ras-GRF1 and Ras-GRF2 in L-DOPA-induced dyskinesia. Ann Clin Transl Neurol 2:662-678.

Borgohain R, Szasz J, Stanzione P, Meshram C, Bhatt MH, Chirilineau D, Stocchi F, Lucini V, Giuliani R, Forrest E, et al.; Study 018 Investigators (2014) Two-year, randomized, controlled study of safinamide as add-on to levodopa in mid to late Parkinson's disease. Mov Disord 29:1273-1280.

Brugnoli A, Napolitano F, Usiello A, and Morari M (2016) Genetic deletion of Rhes or pharmacological blockade of mTORC1 prevent striato-nigral neurons activation in levodopa-induced dyskinesia. Neurobiol Dis 85:155-163.

Caccia C, Maj R, Calabresi M, Maestroni S, Faravelli L, Curatolo L, Salvati P, and Fariello RG (2006) Safinamide: from molecular targets to a new antiParkinson drug. Neurology 67(Suppl 2):S18-S23.

Calabresi P, Pisani A, Rothwell J, Ghiglieri V, Obeso JA, and Picconi B (2016) Hyperkinetic disorders and loss of synaptic downscaling. Nat Neurosci 19:868-875. 
Calon F, Morissette M, Ghribi O, Goulet M, Grondin R, Blanchet PJ, Bédard PJ, and Di Paolo T (2002) Alteration of glutamate receptors in the striatum of dyskinetic 1-methyl-4-phenyl-1,2,3,6-tetrahydropyridine-treated monkeys following dopamine agonist treatment. Prog Neuropsychopharmacol Biol Psychiatry 26:127-138.

Cenci MA, Lee CS, and Björklund A (1998) L-DOPA-induced dyskinesia in the rat is associated with striatal overexpression of prodynorphin- and glutamic acid decarboxylase mRNA. Eur $J$ Neurosci 10:2694-2706.

Cenci MA and Lindgren HS (2007) Advances in understanding L-DOPA-induced dyskinesia. Curr Opin Neurobiol 17:665-671.

Cenci MA and Lundbland M (2007) Ratings of L-DOPA-induced dyskinesia in the unilateral 6-OHDA lesion model of Parkinson's disease in rats and mice. Curr Protoc Neurosci Chapter 9:Unit 9.25.

Dunah AW, Sirianni AC, Fienberg AA, Bastia E, Schwarzschild MA, and Standaert DG (2004) Dopamine D1-dependent trafficking of striatal $N$-methyl-D-aspartate glutamate receptors requires Fyn protein tyrosine kinase but not DARPP-32. Mol Pharmacol 65:121-129.

Dupre KB, Ostock CY, Eskow Jaunarajs KL, Button T, Savage LM, Wolf W, and Bishop C (2011) Local modulation of striatal glutamate efflux by serotonin 1A receptor stimulation in dyskinetic, hemiparkinsonian rats. Exp Neurol 229 288-299.

Errico F, Bonito-Oliva A, Bagetta V, Vitucci D, Romano R, Zianni E, Napolitano F, Marinucci S, Di Luca M, Calabresi P, et al. (2011) Higher free D-aspartate and $N$ methyl-D-aspartate levels prevent striatal depotentiation and anticipate L-DOPAinduced dyskinesia. Exp Neurol 232:240-250.

Gardoni F, Picconi B, Ghiglieri V, Polli F, Bagetta V, Bernardi G, Cattabeni F, Di Luca M, and Calabresi P (2006) A critical interaction between NR2B and MAGUK in L-DOPA induced dyskinesia. J Neurosci 26:2914-2922.

Gardoni F, Sgobio C, Pendolino V, Calabresi P, Di Luca M, and Picconi B (2012) Targeting NR2A-containing NMDA receptors reduces L-DOPA-induced dyskinesias. Neurobiol Aging 33:2138-2144.

Ghiglieri V, Mineo D, Vannelli A, Cacace F, Mancini M, Pendolino V, Napolitano F, di Maio A, Mellone M, Stanic J, et al. (2016) Modulation of serotonergic transmission by eltoprazine in L-DOPA-induced dyskinesia: behavioral, molecular, and synaptic mechanisms. Neurobiol Dis 86:140-153.

Glover V, Elsworth JD, and Sandler M (1980) Dopamine oxidation and its inhibition by (-)-deprenyl in man. J Neural Transm Suppl 16:163-172.

Grégoire L, Jourdain VA, Townsend M, Roach A, and Di Paolo T (2013) Safinamide reduces dyskinesias and prolongs L-DOPA antiparkinsonian effect in parkinsonian monkeys. Parkinsonism Relat Disord 19:508-514.

Gubellini P, Eusebio A, Oueslati A, Melon C, Kerkerian-Le Goff L, and Salin P (2006) Chronic high-frequency stimulation of the subthalamic nucleus and L-DOPA treatment in experimental parkinsonism: effects on motor behaviour and striatal glutamate transmission. Eur $J$ Neurosci 24:1802-1814.

Marti M, Rodi D, Li Q, Guerrini R, Fasano S, Morella I, Tozzi A, Brambilla R, Calabresi P, Simonato M, et al. (2012) Nociceptin/orphanin FQ receptor agonists attenuate L-DOPA-induced dyskinesias. J Neurosci 32:16106-16119.

Marti M, Trapella C, and Morari M (2008) The novel nociceptin/orphanin FQ receptor antagonist Trap-101 alleviates experimental parkinsonism through inhibition of the nigro-thalamic pathway: positive interaction with L-DOPA. $J$ Neurochem 107: $1683-1696$

Mela F, Marti M, Bido S, Cenci MA, and Morari M (2012) In vivo evidence for a differential contribution of striatal and nigral D1 and D2 receptors to L-DOPA induced dyskinesia and the accompanying surge of nigral amino acid levels. Neurobiol Dis 45:573-582.

Mela F, Marti M, Dekundy A, Danysz W, Morari M, and Cenci MA (2007) Antagonism of metabotropic glutamate receptor type 5 attenuates L-DOPA-induced dyskinesia and its molecular and neurochemical correlates in a rat model of Parkinson's disease. J Neurochem 101:483-497.

Mellone M and Gardoni F (2018) Glutamatergic mechanisms in L-DOPA-induced dyskinesia and therapeutic implications. J Neural Transm (Vienna) 125:1225-1236.

Mellone M, Stanic J, Hernandez LF, Iglesias E, Zianni E, Longhi A, Prigent A Picconi B, Calabresi P, Hirsch EC, et al. (2015) NMDA receptor GluN2A/GluN2B subunit ratio as synaptic trait of levodopa-induced dyskinesias: from experimental models to patients. Front Cell Neurosci 9:245

Menegoz M, Lau LF, Hervé D, Huganir RL, and Girault JA (1995) Tyrosine phosphorylation of NMDA receptor in rat striatum: effects of 6-OH-dopamine lesions. Neuroreport 7:125-128.

Morari M, Brugnoli A, Pisanò CA, Novello S, Caccia C, Melloni E, Padoani G, Vailat S, and Sardina M (2018) Safinamide differentially modulates in vivo glutamate and GABA release in the rat hippocampus and basal ganglia. $J$ Pharmacol Exp Ther 364:198-206.

Morari M, O'Connor WT, Ungerstedt U, Bianchi C, and Fuxe K (1996) Functional neuroanatomy of the nigrostriatal and striatonigral pathways as studied with dual probe microdialysis in the awake rat-II. Evidence for striatal $N$-methyl-D-aspartate receptor regulation of striatonigral GABAergic transmission and motor function. Neuroscience 72:89-97.

Morin N, Jourdain VA, and Di Paolo T (2014) Modeling dyskinesia in animal models of Parkinson disease. Exp Neurol 256:105-116

Napolitano M, Picconi B, Centonze D, Bernardi G, Calabresi P, and Gulino A (2006) L-DOPA treatment of parkinsonian rats changes the expression of Src, Lyn and PKC kinases. Neurosci Lett 398:211-214.

Nevalainen N, Lundblad M, Gerhardt GA, and Strömberg I (2013) Striatal glutamate release in L-DOPA-induced dyskinetic animals. PLoS One 8:e55706.

Ostock CY, Dupre KB, Jaunarajs KL, Walters H, George J, Krolewski D, Walker PD, and Bishop C (2011) Role of the primary motor cortex in L-DOPA-induced dyskinesia and its modulation by 5 -HT1A receptor stimulation. Neuropharmacology 61 753-760.

Paillé V, Picconi B, Bagetta V, Ghiglieri V, Sgobio C, Di Filippo M, Viscomi MT, Giampà C, Fusco FR, Gardoni F, et al. (2010) Distinct levels of dopamine denervation differentially alter striatal synaptic plasticity and NMDA receptor subunit composition. J Neurosci 30:14182-14193.

Paolone G, Brugnoli A, Arcuri L, Mercatelli D, and Morari M (2015) Eltoprazine prevents levodopa-induced dyskinesias by reducing striatal glutamate and direct pathway activity. Mov Disord 30:1728-1738.

Paxinos G and Watson C (1986) The Rat Brain in Stereotaxic Coordinates, Academic, Orlando, FL.

Picconi B, Centonze D, Håkansson K, Bernardi G, Greengard P, Fisone G, Cenci MA and Calabresi P (2003) Loss of bidirectional striatal synaptic plasticity in L-DOPAinduced dyskinesia. Nat Neurosci 6:501-506.

Robelet S, Melon C, Guillet B, Salin P, and Kerkerian-Le Goff L (2004) Chronic LDOPA treatment increases extracellular glutamate levels and GLT1 expression in the basal ganglia in a rat model of Parkinson's disease. Eur J Neurosci 20: 1255-1266.

Roche KW, O'Brien RJ, Mammen AL, Bernhardt J, and Huganir RL (1996) Characterization of multiple phosphorylation sites on the AMPA receptor GluR1 subunit. Neuron 16:1179-1188.

Sader-Mazbar O, Loboda Y, Rabey MJ, and Finberg JP (2013) Increased L-DOPAderived dopamine following selective MAO-A or -B inhibition in rat striatum depleted of dopaminergic and serotonergic innervation. $\mathrm{Br} J$ Pharmacol 170 999-1013.

Salvati P, Maj R, Caccia C, Cervini MA, Fornaretto MG, Lamberti E, Pevarello P Skeen GA, White HS, Wolf $\mathrm{HH}$, et al. (1999) Biochemical and electrophysiological studies on the mechanism of action of PNU-151774E, a novel antiepileptic compound. J Pharmacol Exp Ther 288:1151-1159.

Santini E, Sgambato-Faure V, Li Q, Savasta M, Dovero S, Fisone G, and Bezard E (2010) Distinct changes in cAMP and extracellular signal-regulated protein kinase signalling in L-DOPA-induced dyskinesia. PLoS One 5:e12322.

Santini E, Valjent E, Usiello A, Carta M, Borgkvist A, Girault JA, Hervé D, Greengard P, and Fisone G (2007) Critical involvement of cAMP/DARPP-32 and extracellular signal-regulated protein kinase signaling in L-DOPA-induced dyskinesia. $J$ Neurosci 27:6995-7005.

Schapira AH, Fox SH, Hauser RA, Jankovic J, Jost WH, Kenney C, Kulisevsky J, Pahwa R, Poewe W, and Anand R (2017) Assessment of safety and efficacy of safinamide as a levodopa adjunct in patients with Parkinson disease and motor fluctuations: a randomized clinical trial. JAMA Neurol 74:216-224.

Sgambato-Faure V and Cenci MA (2012) Glutamatergic mechanisms in the dyskinesias induced by pharmacological dopamine replacement and deep brain stimulation for the treatment of Parkinson's disease. Prog Neurobiol 96:69-86.

Silverdale MA, Kobylecki C, Hallett PJ, Li Q, Dunah AW, Ravenscroft P, Bezard E, and Brotchie JM (2010) Synaptic recruitment of AMPA glutamate receptor subunits in levodopa-induced dyskinesia in the MPTP-lesioned nonhuman primate. Synapse 64:177-180.

Stanic J, Mellone M, Napolitano F, Racca C, Zianni E, Minocci D, Ghiglieri V, Thiolat ML, Li Q, Longhi A, et al. (2017) Rabphilin 3A: a novel target for the treatment of levodopa-induced dyskinesias. Neurobiol Dis 108:54-64.

Tronci E, Fidalgo C, Zianni E, Collu M, Stancampiano R, Morelli M, Gardoni F, and Carta M (2014) Effect of memantine on L-DOPA-induced dyskinesia in the 6-OHDA-lesioned rat model of Parkinson's disease. Neuroscience 265:245-252.

Youdim MB, Edmondson D, and Tipton KF (2006) The therapeutic potential of monoamine oxidase inhibitors. Nat Rev Neurosci 7:295-309.

Zhang X, Priest BT, Belfer I, and Gold MS (2017) Voltage-gated $\mathrm{Na}^{+}$currents in human dorsal root ganglion neurons. eLife 6:e23235.

Address correspondence to: Dr. F. Gardoni, Department of Pharmacological and Biomolecular Sciences, University of Milan, Via Balzaretti 9, 20133 Milan, Italy. E-mail: fabrizio.gardoni@unimi.it 